\title{
The Extended Bessel-Maitland Function and Integral Operators Associated with Fractional Calculus
}

\author{
Kelelaw Tilahun, Hagos Tadessee, and D. L. Suthar \\ Department of Mathematics, Wollo University, P.O. Box 1145, Dessie, Ethiopia \\ Correspondence should be addressed to D. L. Suthar; dlsuthar@gmail.com
}

Received 25 April 2020; Accepted 29 May 2020; Published 23 June 2020

Academic Editor: Serkan Araci

Copyright (c) 2020 Kelelaw Tilahun et al. This is an open access article distributed under the Creative Commons Attribution License, which permits unrestricted use, distribution, and reproduction in any medium, provided the original work is properly cited.

\begin{abstract}
The aim of this paper is to introduce a presumably and remarkably altered integral operator involving the extended generalized Bessel-Maitland function. Particular properties are considered for the extended generalized Bessel-Maitland function connected with fractional integral and differential operators. The integral operator connected with operators of the fractional calculus is also observed. We point out important links to known findings from some individual cases with our key outcomes.
\end{abstract}

\section{Introduction and Preliminaries}

The Bessel-Maitland function $J_{9}^{\varsigma}($.$) is a generalization of$ Bessel function introduced by Ed. Maitland Wright [1] through a series representation as follows:

$$
J_{\vartheta}^{\varsigma}(z)=\sum_{m=0}^{\infty} \frac{(-z)^{m}}{\Gamma(\varsigma m+\vartheta+1) m !}, \quad(z, \vartheta \in \mathbb{C}, \varsigma>0) .
$$

In fact, Watson's book [2] finds the application of the Bessel-Maitland function in the diverse field of engineering, chemical and biological sciences, and mathematical physics.

Further, Pathak [3] defined generalization of the BesselMaitland function $J_{\vartheta, q}^{\varsigma, \delta}($.$) in the form as follows:$

$$
J_{\vartheta, q}^{\varsigma, \delta}(z)=\sum_{m=0}^{\infty} \frac{(\delta)_{q m}(-z)^{m}}{\Gamma(\varsigma m+\vartheta+1) m !},
$$

where $\quad z \in \mathbb{C} \backslash(-\infty, 0] ; \varsigma, \vartheta, \delta \in \mathbb{C}, \mathfrak{R}(\varsigma) \geq 0, \mathfrak{R}(\vartheta) \geq-1$, $\mathfrak{R}(\delta) \geq 0 ; q \in(0,1) \cup \mathbb{N}$, and $(\delta)_{q m}$ is known as generalized Pochhammer symbol which is defined as

$$
(\delta)_{0}=1,(\delta)_{q m}=\frac{\Gamma(\delta+q m)}{\Gamma(\delta)} .
$$

Since the implementation of the Bessel-Maitland function in 1983, a number of extensions and generalizations have been introduced and examined with different applications (see information in [4-9]).
By motivation of these investigations and applications of the Bessel-Maitland function, Suthar et al. [7] defined the generalized Bessel-Maitland function (2) in the following manner:

$$
\begin{array}{r}
J_{\vartheta, q}^{\zeta, \delta ; c}(z ; p)=\sum_{m=0}^{\infty} \frac{\mathscr{B}_{p}(\delta+q m, c-\delta)(c)_{q m}(-z)^{m}}{B(\delta, c-\delta) \Gamma(c m+\vartheta+1) m !}, \\
(p>0, q \in \mathbb{N}, \mathfrak{R}(c)>\Re(\delta)>0),
\end{array}
$$

which is known as extended generalized Bessel-Maitland function; here, $\mathscr{B}_{p}(s, t)$ is the extended beta function (see [10]).

$$
\begin{array}{r}
\mathscr{B}_{p}(s, t)=\int_{0}^{1} z^{s-1}(1-z)^{t-1} e^{-p / z(1-z)} \mathrm{d} z, \\
(\mathfrak{R}(p)>0, \mathfrak{R}(s)>0, \mathfrak{R}(t)>0) .
\end{array}
$$

For $p=0,(5)$ reduces to beta function (see, e.g., [11], Section 1.1).

Remark 1

(i) The particular case of equation (4), when $p=0$, reduces to (2) and when $p=q=0$, reduces to (1).

(ii) When $q=1, \vartheta=\vartheta-1$ and $z=-z$ in (4) reduce to the extended Mittag-Leffler function defined by Ozarslan and Yilmaz ([12], equation (4)). 
Definition 1. The space of Lebesgue measurable of real or complex valued function $\mathscr{L}(a, b)$ for our study of the significance of fractional calculus is defined as follows:

$$
\mathscr{L}(a, b)=\left(f:\|f\|_{1}=\int_{a}^{b} f(x) \mathrm{d} x<\infty\right) .
$$

Definition 2. The Riemann-Liouville (R-L) fractional integral operators $\mathfrak{\Im}_{a+}^{\ell}$ and $\mathfrak{\Im}_{b-}^{\ell}$ are defined respectively as (see, e.g., [13]) follows:

$$
\begin{aligned}
& \left(\mathfrak{\Im}_{a+}^{\ell} f\right)(x)=\frac{1}{\Gamma(\ell)} \int_{a}^{x}(x-\lambda)^{\ell-1} f(\lambda) \mathrm{d} \lambda, \quad(x>a), \\
& \left(\mathfrak{J}_{b-}^{\ell} f\right)(x)=\frac{1}{\Gamma(\ell)} \int_{x}^{b}(\lambda-x)^{\ell-1} f(\lambda) \mathrm{d} \lambda, \quad(x<b),
\end{aligned}
$$

where $f(x) \in \mathscr{L}(a, b), \ell \in \mathbb{C}$, and $\mathfrak{R}(\ell)>0$.

Definition 3. For $f(x) \in \mathscr{L}(a, b) ; \ell \in \mathbb{C}, \mathfrak{R}(\ell)>0$ and $n=[\Re(\ell)]+1$, the Riemann-Liouville fractional differential operators $\mathscr{D}_{a+}^{\ell}$ are defined by (see, e.g., [13])

$$
\left(\mathscr{D}_{a+}^{\ell} f\right)(x)=\left(\frac{\mathrm{d}}{\mathrm{d} x}\right)^{n}\left(\mathfrak{\Im}_{a+}^{n-\ell} f\right)(x) .
$$

Also, $\mathscr{D}_{a+}^{\ell, v}$ of order $0<\ell<1$ and class $0<v<1$ with reference to $x$ which is the generalized form of (9) (see [13-15]) is defined as follows:

$$
\left(\mathscr{L}_{a+}^{\ell, v} f\right)=\left(\mathfrak{\Im}_{a+}^{v(1-\ell)} \frac{\mathrm{d}}{\mathrm{d} x}\left(\mathfrak{J}_{a+}^{(1-v)(1-\ell)} f\right)\right)(x) .
$$

On setting $v=0$ in (10), it reduces $\mathscr{L}_{a+}^{\ell}$ specified in (9) to the fractional differential operator.

We found the following baseline findings for our study.

Lemma 1 (Mathai and Haubold [16]). If $\ell, u, \in \mathbb{C}, \mathfrak{R}(\ell)>0$, $\mathfrak{R}(u)>0$, then

$$
\left(\mathfrak{J}_{a+}^{\ell}(\lambda-a)^{u-1}\right)(x)=\frac{\Gamma(u)}{\Gamma(\ell+\mu)}(x-a)^{\ell+u-1} .
$$

Lemma 2 (Srivastava and Manocha [17]). If a function $f(z)$ is analytic and has a power series representation $f(z)=\sum_{m=0}^{\infty} a_{m} z^{m}$ in the disc $|z|<\Re$, then

$$
{ }_{0} \mathscr{L}_{z}^{\ell}\left\{z^{u-1} f(z)\right\}=\frac{\Gamma(u)}{\Gamma(\ell+u)} \sum_{m=0}^{\infty} \frac{a_{m}(u)_{m}}{(\ell+u)_{m}} z^{m} .
$$

Lemma 3 (Srivastava and Tomovski [18]). Let $x>a, 0<\ell<1,0 \leq v \leq 1, \mathfrak{R}(\vartheta)>0$ and $\mathfrak{R}(\ell)>0$. Then, the subsequent result holds true for $\mathscr{L}_{a+}^{\ell, v} f$ as follows:

$$
\mathscr{L}_{a+}^{\ell, v}\left[(\lambda-a)^{\vartheta-1}\right](x)=\frac{\Gamma(\vartheta)}{\Gamma(\vartheta-\ell)}(x-a)^{\vartheta-\ell-1} .
$$

We also provided the subsequent established facts and rules in this article.

Fubini's theorem (Dirichlet formula) (Samko et al. [15])

$$
\int_{a}^{b} \mathrm{~d} z \int_{a}^{z} f(z, t) \mathrm{d} t=\int_{a}^{b} \mathrm{~d} t \int_{t}^{b} f(z, t) \mathrm{d} z .
$$

We define the following integral operator in terms of extended generalized Bessel function for $\delta, \omega \in \mathbb{C}, \mathfrak{R}(\varsigma)>0$ and $\mathfrak{R}(\vartheta)>0$ for our further analysis of fractional calculus, then the integral operator

$$
\left(\mathfrak{\Im}_{a+;,, q}^{\omega ; \zeta, \delta ;} f\right)(x)=\int_{a}^{x}(x-\lambda)^{\vartheta} J_{\mathcal{\vartheta}, q}^{\varsigma, \delta ; c}\left(\omega(x-\lambda)^{\varsigma} ; p\right) f(\lambda) \mathrm{d} \lambda,
$$

where $x>$ a.

If we put $p=0$ to the operator, then (15) reduces

$$
\left(\mathfrak{J}_{a+; \vartheta, q}^{\omega ; \zeta, \delta} f\right)(x)=\int_{a}^{x}(x-\lambda)^{\vartheta} J_{\vartheta, q}^{\varsigma, \delta}\left(\omega(x-\lambda)^{\varsigma}\right) f(\lambda) \mathrm{d} \lambda .
$$

If $\omega=0$, then (16) reduces the integral operator to the $\mathrm{R}-\mathrm{L}$ fractional integral operator described in (7).

\section{Integral Operators with Extended Generalized Bessel-Maitland Function in the Kernel}

In this part, we consider the composition of the fractional integral and derivative of Riemann-Liouville and the fractional derivative of Hilfer with the extended generalized Bessel-Maitland function defined by (4).

Theorem 1. Suppose $\varsigma, \vartheta, \delta, c \in \mathbb{C}, \mathfrak{R}(\varsigma)>0, \mathfrak{R}(\vartheta)>-1$, $\mathfrak{R}(c)>\mathfrak{R}(\delta)>0,(p>0)$, and $q, n \in \mathbb{N}$, then the following result holds true:

$$
\left(\frac{\mathrm{d}}{\mathrm{d} z}\right)^{n} z^{\vartheta}\left(J_{\vartheta, q}^{\varsigma, \delta ; c}\left(\omega z^{\varsigma} ; p\right)\right)=z^{\vartheta-n} J_{\vartheta-n, q}^{\varsigma, \delta ; c}\left(\omega z^{\varsigma} ; p\right) .
$$

Proof. Using (4), we see that

$$
\begin{aligned}
\left(\frac{\mathrm{d}}{\mathrm{d} z}\right)^{n} z^{\vartheta}\left(J_{\vartheta, q}^{\varsigma, \delta ; c}\left(\omega z^{\varsigma} ; p\right)\right) & =\left(\frac{\mathrm{d}}{\mathrm{d} z}\right)^{n} z^{\vartheta} \sum_{m=0}^{\infty} \frac{\mathscr{B}_{p}(\delta+q m, c-\delta)(c)_{q m}\left(-\omega z^{\varsigma}\right)^{m}}{B(\delta, c-\delta) \Gamma(\varsigma m+\vartheta+1) m !} \\
& =\sum_{m=0}^{\infty} \frac{\mathscr{B}_{p}(\delta+q m, c-\delta)(c)_{q m}(-\omega)^{m}}{B(\delta, c-\delta) \Gamma(\varsigma m+\vartheta+1) m !}\left(\frac{\mathrm{d}}{\mathrm{d} z}\right)^{n} z^{\varsigma m+\vartheta} .
\end{aligned}
$$


Using the identity,

$$
\left(\frac{\mathrm{d}}{\mathrm{d} x}\right)^{n} x^{m}=\frac{\Gamma(m+1)}{\Gamma(m-n+1)} x^{m-n}, \quad m \geq n,
$$

and after simplifying, we have

$$
\left(\frac{\mathrm{d}}{\mathrm{d} z}\right)^{n} z^{\vartheta}\left(J_{\vartheta, q}^{\varsigma, \delta ; c}\left(\omega z^{\varsigma} ; p\right)\right)=z^{\vartheta-n} \sum_{m=0}^{\infty} \frac{\mathscr{B}_{p}(\delta+q m, c-\delta)(c)_{q m}\left(-\omega z^{\varsigma}\right)^{m}}{B(\delta, c-\delta) \Gamma(\varsigma m+\vartheta-n+1) m !} .
$$

Finally, it can be expressed by using (4) again, and we obtain

$$
\left(\frac{\mathrm{d}}{\mathrm{d} z}\right)^{n} z^{\vartheta}\left(J_{\vartheta, q}^{\varsigma, \delta ; c}\left(\omega z^{\varsigma} ; p\right)\right)=z^{9-n} J_{\vartheta-n, q}^{\varsigma, \delta ; c}\left(\omega z^{\varsigma} ; p\right)
$$

Corollary 1. Suppose $\varsigma, \vartheta, \delta \in \mathbb{C}, \quad \Re(\varsigma)>0, \mathfrak{R}(\vartheta)>-1$, $\Re(\delta)>0, p=0, q \in(0,1) \cup \mathbb{N}$, and $n \in \mathbb{N}$, then the following result holds true:

$$
\left(\frac{\mathrm{d}}{\mathrm{d} z}\right)^{n} z^{\vartheta}\left(J_{\vartheta, q}^{\varsigma, \delta}\left(\omega z^{\varsigma}\right)\right)=z^{9-n} J_{\vartheta-n, q}^{\varsigma, \delta}\left(\omega z^{\varsigma}\right) .
$$

Theorem 2. If $x>a\left(a \in \mathfrak{R}_{+}=(0, \infty)\right), \quad \delta, \ell, \vartheta, \omega \in \mathbb{C}$, $\Re(\vartheta)>-1, \mathfrak{R}(\ell)>0, p>0, q \in \mathbb{N}$, then

$$
\begin{gathered}
\mathcal{J}_{a+}^{\ell}\left[(\lambda-a)^{\vartheta} J_{\vartheta, q}^{\varsigma, \delta ; c}\left(\omega(\lambda-a)^{\varsigma} ; p\right)\right](x) \\
=(x-a)^{\vartheta+\ell} J_{\vartheta+\ell, q}^{\zeta, \delta ; c}\left(\omega(x-a)^{\varsigma} ; p\right), \\
\mathscr{L}_{a+}^{\ell}\left[(\lambda-a)^{9} J_{\vartheta ; q}^{\varsigma, \delta ; c}\left(\omega(\lambda-a)^{\varsigma} ; p\right)\right](x) \\
=(x-a)^{9-\ell} J_{\vartheta-\ell, q}^{\varsigma, \delta ; c}\left(\omega(x-a)^{\varsigma} ; p\right), \\
\mathscr{L}_{a+,}^{\ell, v}\left[(\lambda-a)^{9} J_{\vartheta, q}^{\varsigma, \delta ; c}\left(\omega(\lambda-a)^{\varsigma} ; p\right)\right](x) \\
=(x-a)^{9-\ell} J_{\vartheta-\ell, q}^{\varsigma, \delta ; c}\left(\omega(x-a)^{\varsigma} ; p\right),
\end{gathered}
$$

Proof

(i) Using (4) and (7), we have

$$
\begin{aligned}
& \Im_{a+}^{\ell}\left[(\lambda-a)^{\vartheta} J_{\vartheta, q}^{\varsigma, \delta ; c}\left(\omega(\lambda-a)^{\varsigma} ; p\right)\right](x) \\
& =\frac{1}{\Gamma(\ell)} \int_{a}^{x}(\lambda-a)^{\vartheta} J_{\vartheta, q}^{\varsigma, \delta ; c}\left(\omega(\lambda-a)^{\varsigma} ; p\right)(x-\lambda)^{\ell-1} \mathrm{~d} \lambda \\
& =\sum_{m=0}^{\infty} \frac{\mathscr{B}_{p}(\delta+m q, c-\delta)(c)_{m q}(-\omega)^{m}}{B(\delta, c-\delta) \Gamma(\varsigma m+\vartheta+1) m !} \frac{1}{\Gamma(\ell)}
\end{aligned}
$$

$$
\begin{aligned}
& \cdot \int_{a}^{x}(\lambda-a)^{9+c m}(x-\lambda)^{\ell-1} \mathrm{~d} \lambda \\
&= \sum_{m=0}^{\infty} \frac{\mathscr{B}_{p}(\delta+m q, c-\delta)(c)_{m q}(-\omega)^{m}}{B(\delta, c-\delta) \Gamma(c m+\vartheta+1) m !} \\
& \cdot\left(\mathfrak{J}_{a+}^{\ell}\left[(\lambda-a)^{9+c m}\right]\right)(x) .
\end{aligned}
$$

By use of (11), we have

$$
\begin{aligned}
\mathfrak{J}_{a+}^{\ell} & {\left[(\lambda-a)^{\vartheta} J_{\vartheta, q}^{\varsigma, \delta ; c}\left(\omega(\lambda-a)^{\varsigma} ; p\right)\right](x) } \\
= & \sum_{m=0}^{\infty} \frac{\mathscr{B}_{p}(\delta+m q, c-\delta)(c)_{m q}(-\omega)^{m}}{B(\delta, c-\delta) \Gamma(\varsigma m+\vartheta+1) m !} \frac{\Gamma(c m+\vartheta+1)}{\Gamma(\varsigma m+\vartheta+\ell+1)}(x-a)^{\varsigma m+\vartheta+\ell} \\
= & (x-a)^{\vartheta+\ell} \sum_{m=0}^{\infty} \frac{\mathscr{B}_{p}(\delta+m q, c-\delta)}{B(\delta, c-\delta)} \frac{(c)_{m q}}{\Gamma(\varsigma m+\vartheta+\ell+1)} \frac{\left(-\omega(x-a)^{\varsigma}\right)^{m}}{m !} \\
= & (x-a)^{\vartheta+\ell} J_{\vartheta+\ell, q}^{\varsigma, \delta ; c}\left(\omega(x-a)^{\varsigma} ; p\right) .
\end{aligned}
$$

This occupies in the (23) statement.

(ii) On using (9), we have 


$$
\begin{aligned}
& \mathscr{L}_{a+}^{\ell}\left[(\lambda-a)^{9} J_{\vartheta, q}^{\varsigma, \delta ; c}\left(\omega(\lambda-a)^{\varsigma} ; p\right)\right](x) \\
& \quad=\left(\frac{\mathrm{d}}{\mathrm{d} x}\right)^{n}\left(\mathfrak{J}_{a+}^{n-\ell}\left[(\lambda-a)^{\vartheta} J_{\vartheta, q}^{\varsigma, \delta ; c}\left(\omega(\lambda-a)^{\varsigma} ; p\right)\right]\right)(x),
\end{aligned}
$$

and using (23), this takes the following form:

$$
\begin{aligned}
& \mathscr{L}_{a+}^{\ell}\left[(\lambda-a)^{9} J_{\vartheta, q}^{\varsigma, \delta ; c}\left(\omega(\lambda-a)^{\varsigma} ; p\right)\right](x) \\
& \quad=\left(\frac{\mathrm{d}}{\mathrm{d} x}\right)^{n}\left[(x-a)^{9-\ell+n} J_{\vartheta-\ell+n, q}^{\zeta, \delta c}\left(\omega(x-a)^{\varsigma} ; p\right)\right] .
\end{aligned}
$$

Applying (17), we get

$$
\begin{gathered}
\mathscr{L}_{a+}^{\ell}\left[(\lambda-a)^{\vartheta} J_{\vartheta-\ell, q}^{\varsigma, \delta ; c}\left(\omega(\lambda-a)^{\varsigma} ; p\right)\right](x) \\
=(x-a)^{9-\ell} J_{\zeta, \vartheta-\ell}^{\gamma, q ; c}\left(\omega(x-a)^{\varsigma} ; p\right) .
\end{gathered}
$$

This completes the desired proof (24).

(iii) By using (4), we obtain

$$
\begin{aligned}
\mathscr{L}_{a+}^{\ell, v} & {\left[(\lambda-a)^{\vartheta} J_{\vartheta, q}^{\varsigma, \delta}\left(\omega(\lambda-a)^{\varsigma} ; p\right)\right](x) } \\
& =\left(\mathscr{L}_{a+}^{\ell, v}\left[\sum_{m=0}^{\infty} \frac{\mathscr{B}_{p}(\delta+m q, c-\delta)(c)_{m q}(-\omega)^{m}}{B(\delta, c-\delta) \Gamma(\varsigma m+\vartheta+1) m !}(\lambda-a)^{\varsigma m+\vartheta}\right]\right)(x) \\
& =\sum_{m=0}^{\infty} \frac{\mathscr{B}_{p}(\delta+m q, c-\delta)(c)_{m q}(-\omega)^{m}}{B(\delta, c-\delta) \Gamma(c m+\vartheta+1) m !}\left(\mathscr{L}_{a+}^{\ell, v}\left[(\lambda-a)^{\varsigma m+9}\right]\right)(x) .
\end{aligned}
$$

By applying (13), we get

$$
\begin{aligned}
\mathscr{L}_{a+}^{\ell, v} & {\left[(\lambda-a)^{\vartheta} J_{\vartheta, q}^{\varsigma, \delta, c}\left(\omega(\lambda-a)^{\varsigma} ; p\right)\right](x) } \\
= & \sum_{m=0}^{\infty} \frac{\mathscr{B}_{p}(\delta+m q, c-\delta)(c)_{m q}(-\omega)^{m}}{B(\delta, c-\delta) \Gamma(\varsigma m+\vartheta+1) m !} \frac{\Gamma(c m+\vartheta+1)}{\Gamma(c m+\vartheta-\ell+1)}(x-a)^{\varsigma m+\vartheta-\ell} \\
= & (x-a)^{9-\ell} \sum_{m=0}^{\infty} \frac{\mathscr{B}_{p}(\delta+m q, c-\delta)}{B(\delta, c-\delta)} \frac{(c)_{m q}}{\Gamma(\varsigma m+\vartheta-\ell+1)} \frac{\left(-\omega(x-a)^{\varsigma}\right)^{m}}{m !} \\
& =(x-a)^{9-\ell} J_{\vartheta-\ell, q}^{\varsigma, \delta ; c}\left(\omega(\lambda-a)^{\varsigma} ; p\right),
\end{aligned}
$$

which brings in the necessary proof.

Corollary 2. If $x>a\left(a \in \Re_{+}=(0, \infty)\right), \delta, \ell, \vartheta, \omega \in \mathbb{C}$, $\mathfrak{R}(9)>-1, \mathfrak{R}(\ell)>0, p=0, q \in(0,1) \cup \mathbb{N}$, then Theorem 2 reduces respectively to

$$
\begin{aligned}
& \mathfrak{J}_{a+}^{\ell}\left[(\lambda-a)^{\vartheta} J_{\vartheta, q}^{\varsigma, \delta}\left(\omega(\lambda-a)^{\varsigma}\right)\right](x)=(x-a)^{9+\ell} J_{\vartheta+\ell, q}^{\varsigma, \delta}\left(\omega(x-a)^{\varsigma}\right), \\
& \mathscr{L}_{a+}^{\ell}\left[(\lambda-a)^{\vartheta} J_{\vartheta, q}^{\varsigma, \delta}\left(\omega(\lambda-a)^{\varsigma}\right)\right](x)=(x-a)^{\vartheta-\ell} J_{\vartheta-\ell, q}^{\varsigma, \delta}\left(\omega(x-a)^{\varsigma}\right), \\
& \mathscr{L}_{a+}^{\ell, \nu}\left[(\lambda-a)^{\vartheta} J_{\vartheta, q}^{\varsigma, \delta}\left(\omega(\lambda-a)^{\varsigma}\right)\right](x)=(x-a)^{\vartheta-\ell} J_{\vartheta-\ell, q}^{\varsigma, \delta}\left(\omega(x-a)^{\varsigma}\right) .
\end{aligned}
$$

\section{Some Properties of the \\ Operator $\left(\mathfrak{\Im}_{a+; \vartheta, q}^{\omega ; \zeta, \delta ;} f\right)(x)$}

In this section, we derive several continuity properties of the generalized fractional integral operator.

Theorem 3. If $\delta, \omega \in \mathbb{C}, \mathfrak{R}(\varsigma)>0, \Re(\vartheta)>-1, p>0, q \in \mathbb{N}$, and $\mathfrak{R}(\mu)>0$, then

$$
\left(\Im^{\omega ; ;, \delta ; c}\left[(\lambda-a)^{\mu-1}\right]\right)(x)=(x-a)^{\mu+9} \Gamma(\mu) J_{\vartheta+\mu, q}^{\zeta, \delta ;}\left(\omega(x-a)^{\varsigma} ; p\right) .
$$


Proof. From (4) and (15), we obtain

$$
\begin{aligned}
\left(\Im_{a+;, q}^{\omega ; \zeta, \delta ; c}\left[(\lambda-a)^{\mu-1}\right]\right)(x) & =\int_{a}^{x}(x-\lambda)^{\vartheta}(\lambda-a)^{\mu-1} J_{\vartheta, q}^{\varsigma, \delta ; c}\left(\omega(x-\lambda)^{\varsigma} ; p\right) \mathrm{d} \lambda \\
& =\sum_{m=0}^{\infty} \frac{\mathscr{B}_{p}(\delta+m q, c-\delta)(c)_{m q}(-\omega)^{m}}{B(\delta, c-\delta) \Gamma(\varsigma m+\vartheta+1) m !}\left(\int_{a}^{x}(\lambda-a)^{\mu-1}(x-\lambda)^{\vartheta+\varsigma m} \mathrm{~d} \lambda\right) \\
& =\sum_{m=0}^{\infty} \frac{B_{p}(\delta+m q, c-\delta)(c)_{m q}(-\omega)^{m}}{B(\delta, c-\delta) m !} \frac{1}{\Gamma(\varsigma m+\vartheta+1)} \times \int_{a}^{x}(\lambda-a)^{\mu-1}(x-\lambda)^{\vartheta+\varsigma m} \mathrm{~d} \lambda \\
& =\sum_{m=0}^{\infty} \frac{\mathscr{B}_{p}(\delta+m q, c-\delta)(c)_{m q}(-\omega)^{m}}{B(\delta, c-\delta) m !} \Im_{a+}^{c m+\vartheta+1}\left[(\lambda-a)^{\mu-1}\right](x) \\
& =\sum_{m=0}^{\infty} \frac{\mathscr{B}_{p}(\delta+m q, c-\delta)(c)_{m q}}{B(\delta, c-\delta)} \frac{(-\omega)^{m}}{\Gamma(\mu)} \\
& =(x-a)^{9+\mu} \Gamma(\mu) J_{\vartheta+\mu, q}^{\varsigma, \delta ; c}\left(\omega(x-a)^{\varsigma} ; p\right) .
\end{aligned}
$$

This completes the desired proof.

Corollary 3. If $\delta, \omega \in \mathbb{C}, \mathfrak{R}(\varsigma)>0, \mathfrak{R}(\vartheta)>-1, \mathfrak{R}(\ell)>0$, $q \in(0,1) \cup \mathbb{N}$, and $\mathfrak{R}(\mu)>0$, then

$$
\left(\mathfrak{\Im}_{a+;, q,}^{\omega ; \zeta, \delta}\left[(\lambda-a)^{\mu-1}\right]\right)(x)=(x-a)^{\mu+\vartheta} \Gamma(\mu) J_{\vartheta+\mu, q}^{\varsigma, \delta}\left(\omega(x-a)^{\varsigma}\right) . \quad \text { where }
$$

Theorem 4. If $\delta, \varsigma, \vartheta, \omega, c \in \mathbb{C}, \mathfrak{R}(\varsigma)>0, \mathfrak{R}(\vartheta)>-1$, $\mathfrak{R}(c)>0, p>0$, and $q \in \mathbb{N}$, then

$$
\left\|\Im_{a+; \vartheta, q}^{\omega ; \zeta, \delta, c} \varphi\right\|_{1} \leq \mathcal{S}\|\varphi\|_{1},
$$

$$
\mathcal{S}=(b-a)^{\Re(\vartheta)+1} \sum_{m=0}^{\infty} \frac{\mathscr{B}_{p}(\delta+m q, c-\delta)\left|(c)_{m q}\right|}{B(\delta, c-\delta) \Gamma(\varsigma m+\vartheta+1)(\mathfrak{R}(\vartheta)+\mathfrak{R}(\varsigma) m+1)} \frac{\left|-\omega(b-a)^{\varsigma}\right|^{m}}{m !} .
$$

Proof. From (4), (6), and (15), we have

$$
\begin{aligned}
\left\|\mathfrak{J}_{a+;, q}^{\omega ; \zeta, c} \varphi\right\|_{1} & =\int_{a}^{b}\left|\left(\mathfrak{J}_{a+; \vartheta, q}^{\omega ; \zeta, \delta ; c} \varphi\right)(x)\right| \mathrm{d} x \\
& =\int_{a}^{b}\left|\int_{a}^{x}(x-\lambda)^{\vartheta} J_{\vartheta, q}^{\varsigma, \delta ; c}\left(\omega(x-\lambda)^{\varsigma} ; p\right) \varphi(\lambda) \mathrm{d} \lambda\right| \mathrm{d} x .
\end{aligned}
$$

By exchanging the integration order and using Dirichlet formula (14), we have

$$
\begin{aligned}
\left\|\Im_{a+;, q}^{\omega ; \zeta, \delta ;} \varphi\right\|_{1} \leq & \sum_{m=0}^{\infty} \frac{\mathscr{B}_{p}(\delta+m q, c-\delta)\left|(c)_{m q}\right|\left|(-\omega)^{m}\right|}{B(\delta, c-\delta) \Gamma(\varsigma m+\vartheta+1) m !} \\
& \times \int_{a}^{b}\left[\int_{\lambda}^{b}(x-\lambda)^{\Re(\vartheta)+\Re(\varsigma) m} \mathrm{~d} x\right]|\varphi(\lambda)| \mathrm{d} \lambda .
\end{aligned}
$$

Setting $u=x-\lambda$, we obtain

$$
\left\|\Im_{a+;, q}^{\omega ; \zeta, c} \varphi\right\|_{1} \leq \sum_{m=0}^{\infty} \frac{\mathscr{B}_{p}(\delta+m q, c-\delta)\left|(c)_{m q}\right|\left|(-\omega)^{m}\right|}{B(\delta, c-\delta) \Gamma(\varsigma m+\vartheta+1) m !} \int_{a}^{b}\left[\frac{u^{\Re(9)+\Re(\varsigma) m+1}}{\mathfrak{R}(\vartheta)+\mathfrak{R}(\varsigma) m+1}\right]_{0}^{b-a} \times|\varphi(\lambda)| \mathrm{d} \lambda .
$$

This can also be written as

$$
\left\|\mathfrak{\Im}_{a+;, q, q}^{\omega ; \delta ; c} \varphi\right\|_{1} \leq\left\{(b-a)^{\Re(\vartheta)+1} \sum_{m=0}^{\infty} \frac{B_{p}(\delta+m q, c-\delta)\left|(c)_{m q}\right|\left|\left(-\omega(b-a)^{\varsigma}\right)\right|^{m}}{B(\delta, c-\delta) \Gamma(\varsigma m+\vartheta+1)(\mathfrak{R}(\vartheta)+\mathfrak{R}(\varsigma) m+1) m !}\right\} \cdot \int_{a}^{b}|\varphi(\lambda)| \mathrm{d} \lambda=\delta\|\varphi\|_{1} .
$$

This completes the desired proof. 
Corollary 4. If $\delta, \varsigma, \vartheta, \omega, \in \mathbb{C}, \mathfrak{R}(\varsigma)>0, \mathfrak{R}(\vartheta)>-1$, and where $q \in(0,1) \cup \mathbb{N}$, then

$$
\left\|\mathfrak{\Im}_{a+; \vartheta, q}^{\omega ; \zeta, \delta} \varphi\right\|_{1} \leq \mathfrak{H}\|\varphi\|_{1},
$$

$$
\mathfrak{S}=(b-a)^{\mathfrak{R}(\vartheta)+1} \sum_{m=0}^{\infty} \frac{\left|(\delta)_{m q}\right|}{\Gamma(\varsigma m+\vartheta+1)(\mathfrak{R}(\vartheta)+\mathfrak{R}(\varsigma) m+1)} \frac{\left|-\omega(b-a)^{\varsigma}\right|^{m}}{m !} .
$$

Theorem 5. If $\ell, \delta, \varsigma, \vartheta, \omega \in \mathbb{C}, \mathfrak{R}(\varsigma)>0, \mathfrak{R}(\vartheta)>-1$, $\mathfrak{R}(\delta)>0, \mathfrak{R}(\ell)>0, p>0, q \in \mathbb{N}$, and $x>a$, for any function Proof. From (7) and (15), we have $f \in \mathscr{L}(\varsigma, \vartheta)$, then the result holds true:

$$
\left(\mathfrak{\Im}_{a+}^{\ell}\left[\mathfrak{\Im}_{a+;, q, q}^{\omega ; \zeta, c} f\right]\right)(x)=\left(\mathfrak{\Im}_{a+; \vartheta+\ell, q}^{\omega ; \xi, \delta ; c} f\right)(x)=\left(\mathfrak{J}_{a+;,, q}^{\omega ; \zeta, ; ; c}\left[\mathfrak{J}_{a+}^{\ell} f\right]\right)(x) .
$$

$$
\begin{aligned}
\left(\mathfrak{J}_{a+}^{\ell}\left[\mathfrak{\Im}_{a+;, q}^{\omega ; \zeta, \delta ; c} f\right]\right)(x) & =\frac{1}{\Gamma(\ell)} \int_{a}^{x}(x-\lambda)^{\ell-1}\left[\mathfrak{\Im}_{a+;,, q}^{\omega ; \zeta, \delta} f\right](\lambda) \mathrm{d} \lambda \\
& =\frac{1}{\Gamma(\ell)} \int_{a}^{x}(x-\lambda)^{\ell-1}\left[\int_{a}^{\lambda}(\lambda-u)^{\vartheta} J_{\vartheta, q}^{\zeta, \delta ; c}\left(\omega(\lambda-u)^{\varsigma} ; p\right) f(u) \mathrm{d} u\right] \mathrm{d} \lambda
\end{aligned}
$$

By interchanging the order of integration and using (14), we have

$$
\left(\mathfrak{\Im}_{a+}^{\ell}\left[\mathfrak{J}_{a+;, q, q}^{\omega ; \zeta, \delta ; c} f\right]\right)(x)=\int_{a}^{x}\left[\frac{1}{\Gamma(\ell)} \int_{u}^{x}(x-\lambda)^{\ell-1}(\lambda-u)^{\vartheta} J_{\vartheta, q}^{\varsigma, \delta ; c}\left(\omega(\lambda-u)^{\varsigma} ; p\right) \mathrm{d} \lambda\right] \times f(u) \mathrm{d} u
$$

Setting $\lambda-u=\rho$, we obtain

$$
\left(\mathfrak{\Im}_{a+}^{\ell}\left[\mathfrak{\Im}_{a+; \vartheta, q}^{\omega ; \zeta, \delta ; c} f\right]\right)(x)=\int_{a}^{x}\left[\frac{1}{\Gamma(\ell)} \int_{0}^{x-u}(x-u-\rho)^{\ell-1}(\rho)^{\vartheta} J_{\vartheta, q}^{\varsigma, \delta ; c}\left(\omega \rho^{\varsigma} ; p\right) \mathrm{d} \rho\right] f(u) \mathrm{d} u .
$$

By applying (7) and (23), we get

$$
\left(\mathfrak{J}_{a+}^{\ell}\left[\mathfrak{\Im}_{a+;, \xi, q}^{\omega ; \zeta ; c} f\right]\right)(x)=\int_{a}^{x}\left[(x-u)^{\ell+\vartheta} J_{\vartheta+\ell, q}^{\varsigma, \delta ; c}\left(\omega(x-u)^{\varsigma} ; p\right)\right] f(u) \mathrm{d} u
$$

thus, using (15), we get

$$
\left(\mathfrak{\Im}_{a+}^{\ell}\left[\mathfrak{\Im}_{a+;, q, q}^{\omega ; \zeta, \delta ; c} f\right]\right)(x)=\left(\mathfrak{J}_{a+;, \vartheta+\ell, q}^{\omega ; \zeta, \delta ; c} f\right)(x) .
$$

To demonstrate the second part, we start from the right side of (45), and using (7) and (15), we have 


$$
\begin{aligned}
\left(\mathfrak{J}_{a+;, q, q}^{\omega ; \zeta, \delta ;}\left[\mathfrak{\Im}_{a+}^{\ell} f\right]\right)(x) & =\int_{a}^{x}(x-\lambda)^{\vartheta} J_{\vartheta, q}^{\varsigma, \delta ; c}\left(\omega(x-\lambda)^{\varsigma} ; p\right)\left[\mathfrak{J}_{a+}^{\ell} f\right](\lambda) \mathrm{d} \lambda \\
& =\int_{a}^{x}\left[(x-\lambda)^{\vartheta} J_{\vartheta, q}^{\varsigma, \delta ; c}\left(\omega(x-\lambda)^{\varsigma} ; p\right)\left(\frac{1}{\Gamma(\ell)} \int_{a}^{\lambda}(\lambda-u)^{\ell-1} f(u) \mathrm{d} u\right)\right] \mathrm{d} \lambda \\
& =\int_{a}^{x}\left[\frac{1}{\Gamma(\ell)} \int_{a}^{\lambda}(\lambda-u)^{\ell-1}(x-\lambda)^{\vartheta} J_{\vartheta, q}^{\varsigma, \delta ; c}\left(\omega(x-\lambda)^{\varsigma} ; p\right) f(u) \mathrm{d} u\right] \mathrm{d} \lambda .
\end{aligned}
$$

By interchanging the order of integration and using (14), we obtain

$$
\left(\mathfrak{\Im}_{a+;,, q}^{\omega ; \zeta, \delta ;}\left[\mathfrak{\Im}_{a+}^{\ell} f\right]\right)(x)=\int_{a}^{x} \frac{1}{\Gamma(\ell)}\left[\int_{u}^{x}(x-\lambda)^{\vartheta}(\lambda-u)^{\ell-1} J_{\vartheta, q}^{\varsigma, \delta ; c}\left(\omega(x-\lambda)^{\varsigma} ; p\right) \mathrm{d} \lambda\right] \times f(u) \mathrm{d} u .
$$

Setting $x-\lambda=\rho$, we have

$$
\left(\mathfrak{\Im}_{a+;,, q}^{\omega ; \delta ; c}\left[\mathfrak{J}_{a+}^{\ell} f\right]\right)(x)=\int_{a}^{x} \frac{1}{\Gamma(\ell)}\left[\int_{0}^{x-u} \rho^{\vartheta}(x-u-\rho)^{\ell-1} J_{\vartheta, q}^{\zeta, \delta ; c}\left(\omega \rho^{\varsigma} ; p\right) \mathrm{d} \rho\right] f(u) \mathrm{d} u
$$

Again, by using (7) and applying (23), we get

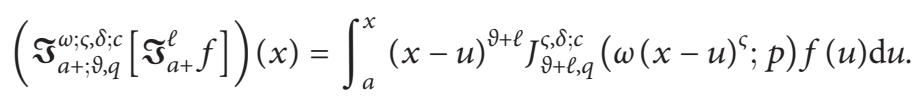

Finally, using (15), we obtain

$$
\left(\mathfrak{\Im}_{a+;, q, q}^{\omega ; \zeta ; c}\left[\mathfrak{\Im}_{a+}^{\ell} f\right]\right)(x)=\left(\mathfrak{\Im}_{a+;,+\ell, \ell, q}^{\omega ; \zeta ; c} f\right)(x) .
$$

Thus, (50) and (55) complete the desired proof of
Corollary 5. If $\ell, \delta, \varsigma, \vartheta, \omega \in \mathbb{C}, \mathfrak{R}(\varsigma)>0, \mathfrak{R}(\vartheta)>-1$, $\mathfrak{R}(\delta)>0, \mathfrak{R}(\ell)>0, q \in(0,1) \cup \mathbb{N}$, and $x>a$, for any function $f \in \mathscr{L}(\varsigma, \vartheta)$, then Theorem 5 takes the form: (45).

$$
\left(\mathfrak{\Im}_{a+}^{\ell}\left[\mathfrak{\Im}_{a+;,, q}^{\omega ; \zeta, \delta} f\right]\right)(x)=\left(\mathfrak{\Im}_{a+; 9+\ell, q}^{\omega ; \zeta, \delta} f\right)(x)=\left(\mathfrak{\Im}_{a+;, q}^{\omega ; \zeta, \delta}\left[\mathfrak{\Im}_{a+}^{\ell} f\right]\right)(x)
$$

\section{Concluding Remark and Discussion}

The newly defined integral operators involving the extended generalized Bessel-Maitland function is investigated here. Various special cases of the paper's related results may be analyzed by taking appropriate values of the relevant parameters. For example, as given in remarks (i) and (ii), we obtain the undeniable result due to Gauhar et al. [19, 20]. For a number of other special cases, we refer to [21] and leave the findings to interested readers.

\section{Data Availability}

No data used to support the study.

\section{Conflicts of Interest}

The authors declare no conflicts of interest.

\section{Authors' Contributions}

All authors contributed equally to the present investigation. All authors read and approved the final manuscript.

\section{References}

[1] O. I. Marichev, Handbook of Integral Transform and Higher Transcendental Functions, John Wiley and Sons, New York, NY, USA, 1983. 
[2] G. N. Watson, A Treatise on the Theory of Bessel Functions, Cambridge University Press, Cambridge, UK, 1965.

[3] R. S. Pathak, "Certain convergence theorems and asymptotic properties of a generalization of Lommel and Maitland transform," ceedings of the National Academy of Sciences, India, Section A, vol. 36, pp. 81-86, 1966.

[4] S. D. Purohit, D. L. Suthar, and S. L. Kalla, "Marichev-SaigoMaeda fractional integration operators of the Bessel functions," Matematiche, vol. 67, no. 1, pp. 12-32, 2012.

[5] D. L. Suthar and H. Amsalu, "Certain integrals associated with the generalized Bessel-Maitland function," Applications and Applied Mathematics, vol. 12, no. 2, pp. 1002-1016, 2017.

[6] D. L. Suthar and H. Habenom, "Integrals involving generalized Bessel-Maitland function," Journal of Science and Arts, vol. 37, no. 4, pp. 357-362, 2016.

[7] D. L. Suthar, A. M. Khan, A. Alaria, S. D. Purohit, and J. Singh, "Extended Bessel-Maitland function and its properties pertaining to integral transforms and fractional calculus," AIMS Mathematics, vol. 5, no. 2, pp. 1400-1410, 2020.

[8] D. L. Suthar, S. D. Purohit, and R. K. Parmar, "Generalized fractional calculus of the multiindex Bessel function," Mathematics in Natural Science, vol. 1, no. 1, pp. 26-32, 2017.

[9] D. L. Suthar, G. V. Reddy, and N. Abeye, "Integral formulas involving product of Srivastava's polynomial and generalized Bessel Maitland functions," International Journal of Science and Research, vol. 11, no. 6, pp. 343-351, 2017.

[10] M. A. Chaudhry, A. Qadir, H. M. Srivastava, and R. B. Paris, "Extended hypergeometric and confluent hypergeometric functions," Applied Mathematics and Computation, vol. 159, no. 2, pp. 589-602, 2004.

[11] H. M. Srivastava and J. Choi, Zeta and Q-Zeta Functions and Associated Series and Integrals, Elsevier Science Publishers, Amsterdam, Netherlands, 2012.

[12] M. A. Zarslan and B. Yilmaz, "The extended Mittag-Leffler function and its properties," Journal of Inequalities and Applications, vol. 2014, no. 1, p. 85, 2014.

[13] A. A. Kilbas, H. M. Srivastava, and J. J. Trujillo, Theory and Applications of Fractional Differential Equations, Elsevier, Amsterdam, Netherlands, 2006.

[14] S. B. Rao, J. C. Prajapati, A. K. Patel, and A. K. Shukla, "Some properties of wright-type hypergeometric function via fractional calculus," Advances in Difference Equations, vol. 2014, p. 119, 2014.

[15] S. G. Samko, A. A. Kilbas, and O. I. Marichev, Fractional Integrals and Derivatives: Theory and Applications, Gordon and Breach Science Publishers, Yverdon, Switzerland, 1993.

[16] A. M. Mathai and H. J. Haubold, Special Functions for Applied Scientists, Springer, Berlin, Germany, 2010.

[17] H. M. Srivastava and H. L. Manocha, A Treatise on Generating Functions, Wiley/Ellis Horwood, New York, NY, USA, 1984.

[18] H. M. Srivastava and Ž. Tomovski, "Fractional calculus with an integral operator containing a generalized Mittag-Leffler function in the kernel," Applied Mathematics and Computation, vol. 211, no. 1, pp. 198-210, 2009.

[19] G. Rahman, P. Agarwal, S. Mubeen, and M. Arshad, "Fractional integral operators involving extended Mittag-Leffler function as its kernel," Boletín de la Sociedad Matemática Mexicana, vol. 24, no. 2, pp. 381-392, 2017.

[20] G. Rahman, D. Baleanu, M. Al Qurashi, S. D. Purohit, S. Mubeen, and M. Arshad, "The extended Mittag-Leffler function via fractional calculus," The Journal of Nonlinear Sciences and Applications, vol. 10, no. 8, pp. 4244-4253, 2017.

[21] T. O. Salim and A. W. Faraj, "A generalization of MittagLeffler function and Integral operator associated with the
Fractional calculus," Journal of Fractional Calculus and Applications, vol. 3, no. 5, pp. 1-13, 2012. 